\title{
Sign Patterns for Eigenmatrices of Nonnegative Matrices
}

\author{
Steve Kirkland* \\ Hamilton Institute \\ National University of Ireland Maynooth \\ Ireland \\ stephen.kirkland@nuim.ie
}

July 14, 2010

\begin{abstract}
For a square $(0,1,-1)$ sign pattern matrix $S$, denote the qualitative class of $S$ by $Q(S)$. In this paper, we investigate the relationship between sign patterns and matrices that diagonalise an irreducible nonnegative matrix. We explicitly describe the sign patterns $S$ such that every matrix in $Q(S)$ diagonalises some irreducible nonnegative matrix. Further, we characterise the sign patterns $S$ such that some member of $Q(S)$ diagonalises an irreducible nonnegative matrix. Finally, we provide necessary and sufficient conditions for a multiset of real numbers to be realised as the spectrum of an irreducible nonnegative matrix $M$ that is diagonalised by a matrix in the qualitative class of some $S^{2} N S$ sign pattern.
\end{abstract}

Keywords Sign pattern; Irreducible nonnegative matrix; Diagonalisation.

AMS Classifications 15A48, 15A18, 05C50

\section{Introduction}

Suppose that $M$ is an irreducible $n \times n$ nonnegative matrix. The celebrated PerronFrobenius theorem [7] asserts that the spectral radius of $M$ is an algebraically simple

*This material is based upon works supported, in part, by the Science Foundation Ireland under Grant No. SFI/07/SK/I1216b. 
eigenvalue, known as the Perron value of $M$. A good deal is also known about the non-Perron eigenvalues of nonnegative matrices. For example, results of Dmitriev and Dynkin [4] and Karpelevich [6] identify, for each $n \geq 2$, the region in the complex plane $\{\lambda \mid \lambda$ is an eigenvalue of an $n \times n$ nonnegative matrix with Perron value 1$\}$. In a similar vein, there has been a great deal of interest in the so-called inverse eigenvalue problem for nonnegative matrices - that is, the problem of determining necessary and sufficient conditions for a multiset of $n$ complex numbers to be the spectrum of a nonnegative matrix of order $n$. We refer the interested reader to [1] and [5] for overviews of the progress on this problem.

The Perron-Frobenius theorem also gives information about the eigenvectors associated with the Perron value of an irreducible nonnegative matrix $M$. Specifically, there is a eigenvector $v$, known as a Perron vector, corresponding to the Perron value of $M$ such that $v$ has all positive entries; further, if $w$ is an eigenvector of $M$ such that all nonzero entries in $w$ have the same complex argument, then necessarily $w$ is a scalar multiple of $v$. Thus we have fairly complete information regarding the sign structure of vectors in the eigenspace associated with the Perron value.

What can be said about the structure of the non-Perron eigenvectors of an irreducible nonnegative matrix? In order to investigate that question, we make the following definition: we say that an $n \times n$ matrix $A$ is a Perron eigenmatrix if there is an irreducible nonnegative matrix of order $n$ that is diagonalised by $A$. In this paper, we consider sign patterns associated with Perron eigenmatrices. It is clear that a Perron eigenmatrix will, in general, contain complex entries; while we will provide some results on the complex case, our principal focus in this paper is on the case that the matrix in question has real entries.

Recall that an $m \times n$ sign pattern matrix $S$ is a $(0,1,-1)$ matrix, and the associated qualitative class $Q(S)$ is given by

$$
Q(S)=\left\{A \in \mathbb{R}^{m \times n} \mid \operatorname{sgn}\left(A_{i j}\right)=\operatorname{sgn}\left(S_{i j}\right), i=1, \ldots, m, j=1, \ldots, n\right\} .
$$

We say that two $m \times n$ matrices $A$ and $B$ have the same sign pattern if both are in $Q(S)$ for some sign pattern matrix $S$. The book [3] contains a wealth of information on sign pattern matrices and their various properties.

For $n \times n$ sign pattern matrices, we consider the following 'require' and 'allow' problems:

- characterise the $n \times n$ sign pattern matrices $S$ such that every matrix in $Q(S)$ is a Perron eigenmatrix;

- characterise the $n \times n$ sign pattern matrices $S$ such that there is an $A \in Q(S)$ that is a Perron eigenmatrix.

In Section 3, both of these problems are solved. In Section 4 we consider the so-called strong sign nonsingular matrices, or $S^{2} N S$ matrices, and determine the multisets of 
real numbers that are realised as the spectrum of an irreducible nonnegative matrix that is diagonalised by a matrix $A$ in the qualitative class of some $S^{2} N S$ sign pattern. Taken together, the results in Sections 2, 3 and 4 can be thought of as addressing an inverse eigenvector problem for nonnegative matrices.

Throughout the paper, we make use of basic notions from nonnegative matrix theory, and from combinatorial matrix theory. We refer the reader to [7] and [2], respectively, for the necessary background material.

\section{Preliminaries}

In this section we develop some basic results that will be useful in the subsequent sections. We begin with a characterisation of Perron eigenmatrices.

Theorem 2.1 Let $A$ be an $n \times n$ matrix with entries in $\mathbb{C}$. For each $i=1, \ldots, n$, let $e_{i}$ denote the $i$-th standard unit basis vector. Then $A$ is a Perron eigenmatrix if and only if both of the following conditions hold:

i) $A$ is invertible;

ii) there is an invertible diagonal matrix $D$ and a permutation matrix $P$ such that for the matrix $B=A D P$, both $B e_{1}$ and $e_{1}^{T} B^{-1}$ have all positive entries.

Proof: Suppose first that $A$ is a Perron eigenmatrix and that $M$ is an irreducible nonnegative matrix diagonalised by $A$. Evidently, $A$ must be invertible. Further, from Perron-Frobenius theory, $M$ has a right eigenvector $v$ with all positive entries, and the only eigenvectors of $M$ having all nonzero entries with the same argument are scalar multiples of $v$. Recall also that if $A e_{i}$ is a right eigenvector of $M$ corresponding to eigenvalue $\lambda$, then $e_{i}^{T} A^{-1}$ is a left eigenvector of $M$ corresponding to $\lambda$. Again using Perron-Frobenius theory, it follows that if $A e_{i}$ has all entries with the same argument, then so does $e_{i}^{T} A^{-1}$. Condition ii) now follows readily.

Next suppose that conditions i) and ii) hold. Observe that for any $n \times n$ matrix $M$, the matrix $A$ diagonalises $M$ if and only if the matrix $B$ does. Thus, it suffices to produce an irreducible nonnegative matrix that is diagonalised by $B$. Consider the matrix $M=B e_{1} e_{1}^{T} B^{-1}$. As both $B e_{1}$ and $e_{1}^{T} B^{-1}$ are positive vectors, $M$ is a positive matrix. Further, $M B=B e_{1} e_{1}^{T}$, and as $e_{1} e_{1}^{T}$ is diagonal, we see that $M$ is diagonlised by $B$, and hence by $A$.

Remark 2.2 Observe that Theorem 2.1 focuses on diagonalisation of irreducible nonnegative matrices. Inspecting the proof of that result, it is not difficult to determine 
that conditions i) and ii) are necessary and sufficient for $A$ to have the property that for some irreducible nonnegative matrix $M, A^{-1} M A$ is in Jordan canonical form. For ease of exposition in the remainder of the paper, we continue to focus on diagonalisation, but we remain mindful of the fact that many of our results also apply to more complicated Jordan structures.

Recall that a square matrix $M$ is said to be partly decomposable if there are permutation matrices $P$ and $Q$ such that $P M Q$ can be written as $P M Q=\left[\begin{array}{l|l}A & 0 \\ \hline B & C\end{array}\right]$, where the blocks $A$ and $C$ are square and nonempty. On the other hand if no such $P$ and $Q$ exist, then $M$ is said to be fully indecomposable. A vector with all real entries is said to be balanced if it is either the zero vector, or has both positive and negative entries; a real vector is unisigned if it not balanced - i.e. it is not the zero vector, and all of its nonzero entries have the same sign.

Proposition 2.3 Suppose that $A$ is an $n \times n$ matrix with entries in $\mathbb{C}$. If $A$ is a Perron eigenmatrix, then the following conditions hold:

i) $A$ is fully indecomposable;

ii) there is an index $j$ between 1 and $n$, a positive vector $x$, and scalars $\alpha, \beta$ not both 0, such that $\operatorname{Re}\left(A e_{j}\right)=\alpha x$ and $\operatorname{Im}\left(A e_{j}\right)=\beta x$;

iii) for each index $l \neq j$, both $\operatorname{Re}\left(A e_{l}\right)$ and $\operatorname{Im}\left(A e_{l}\right)$ are balanced.

Proof: i) Suppose to the contrary that $A$ is partly decomposable and diagonalises the irreducible nonnegative matrix $M$. Then there are permutation matrices $P$ and $Q$ such that $B=P A Q$ has the form $B=\left[\begin{array}{c|c}B_{1} & 0 \\ \hline B_{3} & B_{2}\end{array}\right]$, where the blocks $B_{1}$ and $B_{2}$ are square, say with $B_{1}$ of order $k$, with $1 \leq k \leq n-1$. Note that $B$ is an eigenmatrix for the irreducible nonnegative matrix $P M P^{T}$.

From Perron-Frobenius theory, there is an index $p$ such that $B e_{p}$ has no zero entries, and $e_{p}^{T} B^{-1}$ contains no zero entries. From the former, it must be the case that $1 \leq p \leq k$, while from the latter we find that $k+1 \leq p \leq n$, a contradiction. We conclude that $S$ is fully indecomposable.

ii) From Perron-Frobenius theory, there is a unique index $j$ such that all entries of $A e_{j}$ are nonzero and have the same complex argument, say $\theta \in[0,2 \pi)$. Hence $A e_{j}=\cos (\theta) x+i \sin (\theta) x$ for some positive vector $x$. Setting $\alpha=\cos (\theta)$ and $\beta=\sin (\theta)$ yields the conclusion.

iii) Let $y^{T}$ be a positive left Perron vector for $M$. Then for each index $l \neq j, y^{T} A e_{l}=0$; the conclusions now follow. 
While the focus of this paper is on sign pattern matrices, a Perron eigenmatrix can, in general, contain complex entries. In order to provide a framework for discussing patterns for Perron eigenmatrices with complex entries, we introduce the following terminology.

Given two $n \times n(0,1,-1)$ sign pattern matrices $S$ and $T$, the complex quadrant pattern class for the pair $(S, T)$, which we denote by $Q(S, T)$, is the set of all complex matrices of the form $A_{1} \circ S+i A_{2} \circ T$, where $A_{1}$ and $A_{2}$ are real matrices with all positive entries, and $\circ$ denotes the Hadamard product. Observe that for each pair of indices $k, j=1, \ldots, n$, and every $A \in Q(S, T), A_{k, j}$ lies in the same quadrant of the complex plane as $S_{k, j}+i T_{k, j}$; further, if one or both of $S_{k, j}$ and $T_{k, j}$ is 0 , then $A_{k, j}$ lies on the positive real axis, negative real axis, positive imaginary axis, negative imaginary axis, or at the origin, according as $S_{k, j}+i T_{k, j}$ does.

\section{Require and Allow Results}

In this section we consider the interplay between real Perron eigenmatrices and their corresponding sign patterns. Let $S$ be an $n \times n(0,1,-1)$ sign pattern matrix such that every matrix in $Q(S)$ is a Perron eigenmatrix. Then in particular, every matrix in $Q(S)$ is nonsingular, so that $S$ is, by definition, a sign nonsingular matrix (see [3]). It follows from sign nonsingularity, and from the fact that some column of $S$ has all entries of one sign, that there is a permutation matrix $P$, and a diagonal matrix $D$ with entries 1 or -1 on the diagonal, such that

- $S P D$ has all diagonal entries equal to -1 , and

- the first column of $S D P$ is equal to $\mathbf{- 1}$, where $\mathbf{1}$ denotes the all ones vector.

We refer to this as the standard form for $S$.

Given an $n \times n$ matrix $M$ with real entries, we let $D(M)$ denote its directed graph - i.e. the directed graph on vertices labeled $1, \ldots, n$, such that for each $i, j=$ $1, \ldots, n, D(M)$ contains the arc $i \rightarrow j$ if and only if $M_{i j} \neq 0$. In the case that $i \rightarrow j$ in $D(M)$, we refer to $M_{i j}$ as the weight of the arc $i \rightarrow j$. Given a path (respectively, cycle) in $D(M)$, its sign is the sign of the product of the weights of the arcs on that path (respectively, cycle). From Theorem 3.2.1 of [3], we find that for a $(0,1,-1)$ sign pattern matrix $S$ with all main diagonal entries negative, $S$ is sign nonsingular if and only if the weight of every cycle in $D(S)$ is negative.

The following special subclass of sign nonsingular matrices will play a key role in the first result of this section. An $n \times n(0,1,-1)$ sign pattern matrix $S$ is an $S N S^{*}$ matrix if it is fully indecomposable, sign nonsingular, and has a row each of whose entries is nonzero. Such matrices are discussed in detail in Section 4.2 of [3], where a recursive algorithm is given for determining whether or not a given sign pattern 
matrix is an $\mathrm{SNS}^{*}$-matrix.

Our next result addresses the 'require' problem mentioned in Section 1.

Theorem 3.1 Let $S$ be a $(0,1,-1)$ sign pattern matrix in standard form. Every matrix in $Q(S)$ is a Perron eigenmatrix if and only if $S^{T}$ is an $S N S^{*}$-matrix.

Proof: Suppose first that every $A \in Q(S)$ is a Perron eigenmatrix. Then certainly each such $A$ is invertible, so that $S$ is sign nonsingular. Further, by Proposition 2.3, any $A \in Q(S)$ is fully indecomposable, and hence so is $S$. By hypothesis, the first column of $S$ has no zero entries. It now follows that $S^{T}$ is an SNS*-matrix.

Conversely, suppose that $S^{T}$ is an SNS*-matrix. It then follows that $S$ is both fully indecomposable and sign nonsingular. Since $S_{j 1}=-1$ for each $j=2, \ldots, n$ we find from Theorem 3.2.5 of [3] that for each $A \in Q(S)$, the $(1, j)$ entry of $A^{-1}$ is negative. From the fact that $S$ is sign nonsingular with negative diagonal it follows that for each $A \in Q(S)$, the $(1,1)$ entry of $A^{-1}$ is also negative. From Proposition 2.1 we find that each $A \in Q(S)$ is a Perron eigenmatrix.

Example 3.2 Consider the following $(0,1,-1)$ sign pattern matrix:

$$
S=\left[\begin{array}{cccccc}
-1 & 1 & 1 & 1 & 0 & 0 \\
-1 & -1 & 1 & 0 & 0 & 0 \\
-1 & 0 & -1 & 0 & 0 & 0 \\
-1 & 0 & 0 & -1 & 1 & 1 \\
-1 & 0 & 0 & -1 & -1 & 1 \\
-1 & 0 & 0 & -1 & 0 & -1
\end{array}\right]
$$

The directed graph for $S$ is depicted in Figure 1. It is readily verified that $D(S)$ is strongly connected, and since $S$ has negative main diagonal, it follows from Theorem 4.2.2 of [2] that $S$ is fully indecomposable. Analysing $D(S)$ we find that every cycle contains precisely one arc of negative weight, from which we find that $S$ is sign nonsingular. Consequently, $S^{T}$ is an $\mathrm{SNS}^{*}$-matrix, and so by Theorem 3.1, every matrix in $Q(S)$ is a Perron eigenmatrix.

Alternatively, one can apply the algorithm for recognising an SNS*-matrix given in Section 4.2 of [3] in order to determine that $S^{T}$ is an $\mathrm{SNS}^{*}$-matrix, and hence that every matrix in $Q(S)$ is a Perron eigenmatrix. 


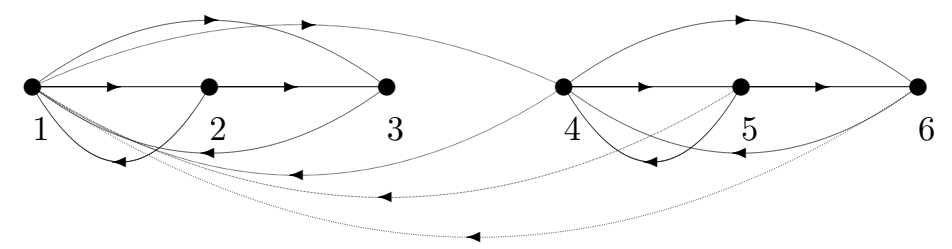

Figure 1: Digraph for Example 3.2 with loops not shown; arcs oriented from left to right have positive signs, while arcs oriented from right to left have negative signs.

Our next result addresses the question of determining which quadrant pattern classes require each matrix in the class to be a Perron eigenmatrix. It turns out that this question can be discussed in terms of the corresponding question for sign pattern classes.

Proposition 3.3 Suppose that $S$ and $T$ are $n \times n(0,1,-1)$ sign pattern matrices, and consider the corresponding quadrant pattern class $Q(S, T)$. Every matrix in $Q(S, T)$ is a Perron eigenmatrix if and only if the following two conditions hold:

i) for each $j=1, \ldots, n$, one of $S e_{j}$ and $T e_{j}$ is the zero vector; and

ii) every matrix in the sign pattern class $Q(S+T)$ is a Perron eigenmatrix.

Proof: We begin by remarking that if i) holds, then the sign pattern matrix $S+T$ is unambiguously defined, since for each $j=1, \ldots, n,(S+T) e_{j}$ coincides with one of $S e_{j}$ and $T e_{j}$.

Suppose first that i) and ii) hold. From i) it follows that there is a diagonal matrix $D$ with entries 1 or $-i$ on the diagonal such that for each $A \in Q(S, T)$, the matrix $A D$ is has all real entries; further, it is readily seen that $A D \in Q(S+T)$. Applying condition ii), we find that for any $A \in Q(S, T)$, the matrix $A D$ is a Perron eigenmatrix. Hence $A$ is also a Perron eigenmatrix.

Next, we suppose that each matrix in $Q(S, T)$ is a Perron eigenmatrix. Fix an $A \in Q(S, T)$, and note that there is a unique index $j$ and a parameter $\theta \in[0,2 \pi)$ such that for some pair of positive vectors $x, y$ we have $A e_{j}=e^{i \theta} x$ and $e_{j}^{T} A^{-1}=e^{-i \theta} y^{T}$. Fix an index $l \neq j$, and suppose that there are indices $k, m$ (not necessarily distinct) such that $T_{k, l} \neq 0$ and $S_{m, l} \neq 0$.

Since $T_{k, l} \neq 0$, we see that for all $t \in \mathbb{R}$ of sufficiently small absolute value, $A+i t e_{k} e_{l}^{T} \in Q(S, T)$, and $\left(A+i t e_{k} e_{l}^{T}\right)^{-1}=A^{-1}-\frac{i t}{1+i t e_{l}^{T} A^{-1} e_{k}} A^{-1} e_{k} e_{l}^{T} A^{-1}$. Hence, $A+i t e_{k} e_{l}^{T}$ is a Perron eigenmatrix for all sufficiently small $t$, and since $\left(A+i t e_{k} e_{l}^{T}\right) e_{j}=$ $e^{i \theta} x$, we deduce that for all such $t$, all entries of $e_{j}^{T}\left(A+i t e_{k} e_{l}^{T}\right)^{-1}$ have complex argument $-\theta$. Thus, for each real $t$ of sufficiently small absolute value, there is a positive 
vector $z$ (whose entries depend on $t$ ) such that $e_{j}^{T}\left(A^{-1}-\frac{i t}{1+i t e_{l}^{T} A^{-1} e_{k}} A^{-1} e_{k} e_{l}^{T} A^{-1}\right)=$ $e^{-i \theta} z^{T}$. This last is equivalent to $y^{T}-\frac{i t y_{k}}{1+i t e_{1}^{T} A^{-1} e_{k}} e_{l}^{T} A^{-1}=z^{T}$. Note that $z_{k}=$ $\frac{y_{k}}{1+i t e_{l}^{T} A^{-1} e_{k}}$, and since $z_{k}>0$, we see that $\frac{y_{k}}{1+i t e_{l}^{T} A^{-1} e_{k}}$ is a positive real number for all sufficiently small $t$; it now follows that $e_{l}^{T} A^{-1} e_{k}=i r$ for some $r \in \mathbb{R}$. Consequently, we find that $y^{T}-\frac{i t y_{k}}{1-t r} e_{l}^{T} A^{-1}$ is a positive vector for all sufficiently small $t$. Thus, it must be the case that every nonzero entry of $e_{l}^{T} A^{-1}$ is purely imaginary.

Next, we apply the condition that $S_{m, l} \neq 0$. As above, for all real $t$ of sufficiently small absolute value, $A+t e_{m} e_{l}^{T} \in Q(S, T)$ and $\left(A+t e_{m} e_{l}^{T}\right)^{-1}=A^{-1}-$ $\frac{t}{1+t e_{l}^{T} A^{-1} e_{m}} A^{-1} e_{m} e_{l}^{T} A^{-1}$. An argument analogous to the one above reveals that $\frac{y_{m}}{1+t e_{l}^{T} A^{-1} e_{m}}$ is a positive real number for all sufficiently small $t$, so that necessarily $e_{l}^{T} A^{-1} e_{m} \in \mathbb{R}$. We then conclude that each entry of $e_{l}^{T} A^{-1}$ must be real, a contradiction to our earlier conclusion that the entries of $e_{l}^{T} A^{-1}$ are purely imaginary. We thus conclude that for each index $l \neq j$, it must be the case that either $T e_{l}$ is the zero vector, or $S e_{l}$ is the zero vector.

Lastly, suppose that there are indices $m, k$ such that $T_{k, j} \neq 0$ and $S_{m, j} \neq 0$, and consider the matrices $A+i t e_{k} e_{j}^{T}$ and $A+t e_{m} e_{j}^{T}$, both of which are in $Q(S, T)$ for all sufficiently small $t \in \mathbb{R}$. For each index $p \neq j$ we have $\left(A+i e_{k} e_{j}^{T}\right) e_{p}=A e_{p}=$ $\left(A+t e_{m} e_{j}^{T}\right)$; as each such $A e_{p}$ contains entries of differing complex arguments, it must be the case that for all sufficiently small $t$, all entries of $\left(A+i t e_{k} e_{j}^{T}\right) e_{j}=A e_{j}+i t e_{k}$ are nonzero, and have the same complex argument. It now follows that all entries of $A e_{j}$ must be purely imaginary. Applying an analogous argument to $\left(A+t e_{m} e_{j}^{T}\right)=$ $A e_{j}+t e_{m}$, we find that all entries of $A e_{j}$ are real, a contradiction. We conclude that one of $S e_{j}$ and $T e_{j}$ must be the zero vector. This establishes condition i) (and so, as noted above, $S+T$ is a well-defined sign pattern matrix).

Now suppose that $B \in Q(S+T)$. From condition i), we find that there is a diagonal matrix $D$ with entries 1 or $i$ on the diagonal such that $B D \in Q(S, T)$. Thus $B D$ is a Perron eigenmatrix, from which it follows that $B$ itself is a Perron eigenmatrix. Hence condition ii) holds.

The following is immediate from Theorem 3.1 and Proposition 3.3.

Corollary 3.4 Let $S$ and $T$ be $n \times n(0,1,-1)$ sign pattern matrices. Every matrix in $Q(S, T)$ is a Perron eigenmatrix if and only if the following conditions hold:

i) for each $j=1, \ldots, n$, one of $S e_{j}$ and $T e_{j}$ is the zero vector;

ii) some column of $S+T$ is either $\mathbf{1}$ or $\mathbf{- 1}$; and

iii) $(S+T)^{T}$ is an $S N S^{*}$ matrix. 
We now address the 'allow' problem raised in Section 1.

Theorem 3.5 Let $S$ be a $(0,1,-1)$ sign pattern. There is an element of $Q(S)$ that is a Perron eigenmatrix if and only if:

a) $S$ is fully indecomposable; and

b) S contains a unique unisigned column, which necessarily contains no zero entries.

Proof: Suppose that there is an $A \in Q(S)$ that diagonalises an irreducible nonnegative matrix. By Proposition 2.3 $A$ is fully indecomposable, and hence so is $S$. Condition b) follows immediately from Perron-Frobenius theory.

Next, we suppose that a) and b) hold; without loss of generality, we assume that $S e_{1}=-\mathbf{1}$. First we claim that there is a subpattern $\tilde{S}$ of $S$ (i.e. a sign pattern formed by setting some of the nonzero entries in $S$ to zero) such that $\tilde{S} e_{1}=-\mathbf{1}$, and the submatrix of $\tilde{S}$ consisting of its last $n-1$ columns is the oriented incidence matrix of a tree on $n$ vertices (see Section 2.3 of [2] for the definition and basics on oriented incidence matrices). In order to establish the claim, we proceed by induction on $n$, and note that the case $n=2$ is immediate. Let $S$ be a sign pattern of order $n \geq 3$ satisfying conditions a) and b). Without loss of generality, we take $S e_{1}=-\mathbf{1}$. From Corollary 4.2 .4 of [3], $S$ is irreducible, and its zero-nonzero pattern dominates the pattern of at least one permutation matrix of order $n$; we take $S_{i i}=-1, i=1, \ldots, n$, again without loss of generality. Suppose that in $D(S)$, vertex $n$ is at maximum distance from vertex 1 . It then follows that for each $j=2, \ldots, n-1$, there is a shortest path from 1 to $j$ that does not pass through $n$. Hence the subgraph of $D(S)$ induced by vertices $1, \ldots, n-1$ is irreducible, and since $S$ has all nonzero entries on the diagonal, we find that the principal submatrix of $S$ on rows and columns $1, \ldots, n-1$ is fully indecomposable.

Write $S$ in partitioned form as

$$
S=\left[\begin{array}{c|c|c}
\Sigma_{11} & \Sigma_{12} & \Sigma_{13} \\
\hline \Sigma_{21} & \Sigma_{22} & \Sigma_{23} \\
\hline \Sigma_{31} & \Sigma_{32} & -1
\end{array}\right],
$$

where the last diagonal block is $1 \times 1$, where $\left[\frac{\Sigma_{12}}{\Sigma_{22}}\right]$ consists of only unisigned columns (each entry of which is necessarily nonpositive), and where in $\left[\frac{\Sigma_{11}}{\Sigma_{21}}\right]$, all columns after the first have both positive and negative entries. (Here we admit the possibility that the second part of the partitioning may be empty; by a slight abuse of notation, we denote that possibility by $\Sigma_{22}=\emptyset$.) Note that from condition b), 
we necessarily have that $\left[\frac{\Sigma_{13}}{\Sigma_{23}}\right]$ contains at least one 1 , say in position $i$; further if $\Sigma_{22} \neq \emptyset$, we must also have $\Sigma_{32}=\mathbf{1}^{T}$.

First suppose that $\Sigma_{22}=\emptyset$. Then $\Sigma_{11}$ satisfies the induction hypothesis, so it has a subpattern $\tilde{\Sigma}_{11}$ whose last $n-2$ columns form the oriented incidence matrix of a tree, and whose first column is $\mathbf{- 1}$. It now follows readily that the subpattern of $S$ given by $\tilde{S}=\left[\begin{array}{c|c}\tilde{\Sigma}_{11} & e_{i} \\ \hline-e_{1}^{T} & -1\end{array}\right]$ has the desired properties. (Observe that the last $n-1$ columns of $\tilde{S}$ comprise the oriented incidence matrix of a tree formed as follows: take the oriented tree associated with $\tilde{\Sigma}_{11}$ and add a directed arc from vertex $i$ to vertex n.)

Now we suppose that $\Sigma_{22} \neq \emptyset$. Observe that each column of $\left[\begin{array}{c}\Sigma_{12} \\ \Sigma_{22}\end{array}\right]$ contains at least two $-1 \mathrm{~s}$, one of which lies on the diagonal of $S$ (as we have already noted above), and the other off the diagonal of $S$ (otherwise $S$ fails to be fully indecomposable). Consider the pattern $\hat{S}=\left[\begin{array}{c|c}\Sigma_{11} & \hat{\Sigma}_{12} \\ \hline \Sigma_{21} & \hat{\Sigma}_{22}\end{array}\right]$, where $\left[\begin{array}{c}\hat{\Sigma}_{12} \\ \hline \hat{\Sigma}_{22}\end{array}\right]$ is formed from $\left[\begin{array}{c}\Sigma_{12} \\ \hline \Sigma_{22}\end{array}\right]$ by setting its $i$ th row equal to $\mathbf{1}^{T}$. It follows that $\hat{S}$ satisfies the induction hypothesis, and so there is a subpattern $S_{0}$ of $\hat{S}$ whose first column is $-\mathbf{1}$ and whose last $n-2$ columns form the oriented incidence matrix of a tree on $n-1$ vertices. Write $e_{i}^{T} S_{0}$ as $e_{i}^{T} S_{0}=\left[v_{1}^{T} \mid v_{2}^{T}\right]$, where the partitioning is conformal with $\hat{S}$. Next, write $v_{2}^{T}=u^{T}-w^{T}$, where both $u^{T}$ and $w^{T}$ are $(0,1)$ vectors such that $u^{T} w=0$; thus the nonzero entries of $u^{T}$ and $w^{T}$ correspond to $1 \mathrm{~s}$ and $-1 \mathrm{~s}$ in $e_{i}^{T} S_{0}$, respectively. Next, we form $S_{1}$ from $S_{0}$ by replacing its $i$ th row by $\left[v_{1}^{T} \mid-w^{T}\right]$. Finally, we construct the subpattern $\tilde{S}$ of $S$ given by $\tilde{S}=\left[\begin{array}{c|c}S_{1} & e_{i} \\ \hline z^{T} & -1\end{array}\right]$, where $z^{T}$, partitioned conformally with $\hat{S}$, is given by $z^{T}=\left[-e_{1}^{T} \mid u^{T}\right]$.

Observe that the last $n-1$ columns of $\tilde{S}$ comprise the oriented incidence matrix of a tree formed as follows: take the oriented tree associated with $S_{0}$, add the directed arc $i \rightarrow n$, and for each vertex $l$ in the second subset of the partition of $S_{0}$ such that $i \rightarrow l$, replace the directed arc $i \rightarrow l$ by the directed arc $n \rightarrow l$. It now follows that $\tilde{S}$ has the desired properties, completing the proof of the claim.

Now we consider a subpattern $\tilde{S}$ of $S$ whose first columns is $\mathbf{- 1}$ and whose submatrix on the last $n-1$ columns is the oriented incidence matrix of a tree. Let $M$ be the $(0,1,-1)$ matrix in $Q(\tilde{S})$, considered as a matrix with real entries, not as a sign pattern matrix. Note that $M$ can be written as $M=\left[\begin{array}{c|c}-1 & -\mathbf{1}^{T} A \\ \hline-\mathbf{1} & A\end{array}\right]$, where $A$ has 
rank $n-1$. Let $J$ denote an all ones matrix. Observe that $\operatorname{det}(M)=-\operatorname{det}(A+J A)=$ $-\operatorname{det}(I+J) \operatorname{det}(A)=-n \operatorname{det}(A)$. As $A$ has rank $n-1$, we deduce that $M$ is nonsingular. Further, $-\frac{1}{n} \mathbf{1}^{T} M=e_{1}^{T}$, from which we conclude that $e_{1}^{T} M^{-1}=-\frac{1}{n} \mathbf{1}^{T}$. It now follows that we may perturb $M$ slightly to produce an invertible matrix $\hat{M} \in Q(S)$ such that $e_{1}^{T} \hat{M}^{-1}<0^{T}$. Thus, $Q(S)$ contains a Perron eigenmatrix by Proposition 2.1 .

Example 3.6 Consider the $(0,1,-1)$ sign pattern $S$ given by

$$
S=\left[\begin{array}{cccc}
-1 & -1 & 0 & 0 \\
-1 & -1 & -1 & 1 \\
-1 & 1 & -1 & -1 \\
-1 & 0 & 1 & -1
\end{array}\right]
$$

Evidently $S$ satisfies conditions i) and ii) of Theorem 3.5, so that there is some matrix in $Q(S)$ that is a Perron eigenmatrix. As an example of such a matrix, consider $M=\left[\begin{array}{cccc}-1 & -1 & 0 & 0 \\ -1 & -1 & -1 & 1 \\ -1 & 3 & -1 & -1 \\ -1 & 0 & 3 & -1\end{array}\right]$, which is in $Q(S)$. We have

$$
M^{-1}=\frac{1}{10}\left[\begin{array}{cccc}
-2 & -4 & -2 & -2 \\
-8 & 4 & 2 & 2 \\
-6 & 3 & -1 & 4 \\
-16 & 13 & -1 & 4
\end{array}\right] \text {, }
$$

so that by Theorem 2.1, $M$ is a Perron eigenmatrix.

Given a $(0,1,-1)$ sign pattern matrix $S$, we let $|S|$ denote the $(0,1)$ sign pattern formed from $S$ by replacing each entry by its absolute value. The following is immediate from Proposition 2.3.

Proposition 3.7 Suppose that $S$ and $T$ are $n \times n(0,1,-1)$ sign pattern matrices, and consider the corresponding quadrant pattern class $Q(S, T)$. If $Q(S, T)$ contains a Perron eigenmatrix, then the following conditions hold:

i) $|S|+|T|$ is fully indecomposable;

ii) there is an index $j$ between 1 and $n$ and numbers $a, b \in\{1,0,-1\}$ not both zero such that $S e_{j}=a \mathbf{1}$ and $T e_{j}=b \mathbf{1}$;

iii) for each index $l \neq j$, both $S e_{l}$ and $T e_{l}$ are balanced.

In the special case that $T=0$, so that $Q(S, T)=Q(S)$, we find from Theorem 3.5 that conditions i)-iii) of Proposition 3.7 are also sufficient for $Q(S, T)$ to contain 
a Perron eigenmatrix. However, if $T \neq 0$, the following example shows that those conditions are not sufficient for $Q(S, T)$ to contain a Perron eigenmatrix.

Example 3.8 Consider the sign pattern matrices

$$
S=\left[\begin{array}{cccc}
1 & -1 & 0 & 0 \\
1 & 1 & 0 & 0 \\
1 & 0 & 1 & -1 \\
1 & 0 & -1 & 1
\end{array}\right], T=\left[\begin{array}{cccc}
0 & 0 & 1 & 0 \\
0 & 0 & -1 & 0 \\
0 & 0 & 0 & 0 \\
0 & 0 & 0 & 0
\end{array}\right]
$$

Observe that $|S|+|T|$ is fully indecomposable, since it has positive diagonal and is irreducible. Note also that for each $j=2,3,4$, each $S e_{j}$ and $T e_{j}$ is balanced. Evidently $S e_{1}=\mathbf{1}$ while $T e_{1}$ is the zero vector.

We claim that $Q(S, T)$ does not contain any Perron eigenmatrices. To see the claim, suppose that $A \in Q(S, T)$ and that there is a positive vector $y^{T}$ such that $y^{T} A=e_{1}^{T}$. (Observe that were $A$ to be a Perron eigenmatrix, such a $y^{T}$ would necessarily exist.) Using the fact that $y^{T} A e_{j}=0, j=2,3,4$, it now follows that there are positive scalars $\alpha, \beta, \gamma, \delta$ such that $A$ has the form

$$
A=\left[\begin{array}{cccc}
A_{11} & -\alpha y_{2} & i \beta y_{2} & 0 \\
A_{21} & \alpha y_{1} & -i \beta y_{1} & 0 \\
A_{31} & 0 & \gamma y_{4} & -\delta y_{4} \\
A_{41} & 0 & -\gamma y_{3} & \delta y_{3}
\end{array}\right]
$$

Observe then that $A$ is singular, since $\left[\begin{array}{c}0 \\ \beta \delta i \\ \alpha \delta \\ \gamma \alpha\end{array}\right]$ is a null vector for $A$.

We conclude that if $A \in Q(S, T)$ is an invertible matrix, then $e_{1}^{T} A^{-1}$ cannot have all positive entries, and hence that $A$ cannot be a Perron eigenmatrix.

Open Problem 3.9 Find necessary and sufficient conditions for a quadrant pattern class $Q(S, T)$ to contain a Perron eigenmatrix.

\section{The $S^{2} N S$ Case}

In this section, we focus on a special class of sign patterns, the $S^{2} N S$ sign patterns: a square $(0,1,-1)$ sign pattern $S$ is said to be $S^{2} N S$ provided that for every $A \in Q(S)$, $A$ is invertible, and in addition, each matrix in $\left\{A^{-1} \mid A \in Q(S)\right\}$ has the same sign 
pattern. See [3] for background and results on that class of sign patterns. It follows from Theorem 2.1 that if $S$ is an $S^{2} N S$ sign pattern, then $Q(S)$ contains a Perron eigenmatrix if and only if every matrix in $Q(S)$ is a Perron eigenmatrix.

The following definition will be helpful in the sequel. An arborescence with root vertex $v$ is an oriented tree with the property that there is a distinguished vertex $v$ such that for each vertex $u$, there is a directed path from $v$ to $u$.

We begin with a characterisation of $S^{2} N S$ sign pattern classes that allow a Perron eigenmatrix.

Lemma 4.1 Let $S$ be an $S^{2} N S$ sign pattern of order $n$ whose first column is $\mathbf{- 1}$, and whose diagonal entries are all -1 . Then $e_{1}^{T} A^{-1}<0^{T}$ for all $A \in Q(S)$ if and only if there is an oriented tree $T$ on vertices $1, \ldots, n$ such that:

i) for each vertex $j=2, \ldots, n$, there is a directed path in $T$ from 1 to $j$;

ii) for each $j=2, \ldots, n$ and $i=1, \ldots, n, S_{i j}=1$ if and only if $i \rightarrow j$ in $T$;

iii) the only negative entries of $S$ are in the first column and on the diagonal.

Proof: From Theorem 7.1.7 of [3], it follows that $S$ is an $S^{2} N S$ matrix with a full first column if and only the submatrix of $S$ on columns $2, \ldots, n$ is the oriented incidence matrix of a tree. Further, in that case, by Theorem 3.2.5 of [3], for each $A \in Q(S)$, and any pair of index $j=2, \ldots, n$, the sign of $A_{1 j}^{-1}$ is minus the sign of any path from 1 to $j$ in $D(S)$. The conclusions now follow readily.

In the context of Lemma 4.1, we refer to $T$ as the arborescence associated with $S$. The next result provides a convenient normalisation for the sign patterns described in Lemma 4.1.

Lemma 4.2 Let $S$ be an $S^{2} N S$ sign pattern of order $n$ whose first column is negative, and whose diagonal is negative. Then for each $A \in Q(S)$ there are positive diagonal matrices $D_{1}, D_{2}$ such that $D_{1} A D_{2}$ has first column equal to $\mathbf{- 1}$ and each diagonal entry equal to -1 .

Proof: Fix $A \in Q(S)$, let

$$
D_{1}=\left[\begin{array}{ccc}
\frac{1}{\left|A_{11}\right|} & & \\
& \ddots & \\
& & \frac{1}{\left|A_{n n}\right|}
\end{array}\right] \text {, and } D_{2}=\left[\begin{array}{cccc}
1 & & & \\
& \frac{\left|A_{21}\right|}{\left|A_{22}\right|} & & \\
& & \ldots & \\
& & \frac{\left|A_{n 1}\right|}{\left|A_{n n}\right|}
\end{array}\right] .
$$

A computation now suffices to yield the conclusion. 
Suppose that $S$ is an $S^{2} N S$ sign pattern of order $n$, and that conditions i)-iii) of Lemma 4.1 hold. Suppose further that we have a matrix $A \in Q(S)$ whose first column and diagonal consist entirely of -1 s. Then we will say that such a matrix $A$ is in $S^{2} N S$ normalised form. When $A \in Q(S)$ is in $S^{2} N S$ normalised form, for each $j=2, \ldots, n$, we let $a_{j}$ denote the single positive entry of $A$ in its $j$-th column. Evidently $A_{i j}=a_{j}$ if and only if $i \rightarrow j$ in the arborescence associated with $S$, and we refer to $a_{j}$ as the weight of the arc $i \rightarrow j$ in $D(A)$. It is straightforward to show that if $A \in Q(S)$ is in $S^{2} N S$ normalised form, then $e_{1}^{T} A^{-1}$ is a negative scalar multiple of the vector $w^{T}=\left[\begin{array}{llll}1 & p_{12} & \ldots & p_{1 n}\end{array}\right]$, where for each $j=2, \ldots, n, p_{1 j}$ is the product of the weights of the arcs on the path from 1 to $j$ in $D(A)$; by convention, we take $p_{11}=1$. Evidently if $M$ is an irreducible nonnegative matrix that is diagonalised by $A$, then $w^{T}$ is a left Perron vector for $M$.

The next result describes the structure of a nonnegative matrix with real spectrum that is diagonalised by a matrix in $S^{2} N S$ normalised form. We note here that unlike many of our earlier results, the hypothesis that our nonnegative matrix can be brought to diagonal form - as opposed to a more complicated Jordan form - by a matrix in $S^{2} N S$ normalised form, is a key component in establishing (1) below.

Proposition 4.3 Let $S$ be an $S^{2} N S$ sign pattern of order $n$ such that $S e_{1}=-\mathbf{1}$ and $S_{i i}=-1, i=1, \ldots, n$. Suppose that $e_{1}^{T} A^{-1}<0^{T}$ for each $A \in Q(S)$, and denote the arborescence associated with $S$ by $T$. Fix $A \in Q(S)$ and suppose that $A$ is in $S^{2} N S$ normalised form, with arc weights $a_{2}, \ldots, a_{n}$. For each pair of vertices $i, j=1, \ldots, n$, define $p_{i j}$ to be the product of the weights of the arcs on the directed path in $T$ from $i$ to $j$ if such a path exists (which we take to be 1 if $i=j$ ) and define $p_{i j}$ to be 0 if no such directed path exists. Let $M$ be an $n \times n$ matrix that is diagonalised by $A$, with real eigenvalues $1, \lambda_{2}, \ldots, \lambda_{n}$ and corresponding eigenvectors $A e_{1}, A e_{2}, \ldots, A e_{n}$, respectively. Set $m=M e_{1}$. Then we may write $M$ as

$$
M=m\left[\begin{array}{llll}
1 & p_{12} & \ldots & p_{1 n}
\end{array}\right]+\sum_{j=2}^{n} \lambda_{j} E_{j},
$$

where for each $j=2, \ldots, n$, with $i_{j} \rightarrow j$ in $T$, we have

$$
E_{j}=\left(-a_{j} e_{i_{j}}+e_{j}\right)\left[\begin{array}{llll}
p_{j 1} & p_{j 2} & \ldots & p_{j n}
\end{array}\right] .
$$

Proof: We prove (1) by considering both sides of the equation, column by column. For each $j=1, \ldots, n$, let $d(1, j)$ denote the distance from vertex 1 to vertex $j$ in $T$. Fix an index $k=1, \ldots, n$, with $d(1, k)=d$ in $T$. We claim by induction on $d$ that $M e_{k}=p_{1 k} m+\sum_{j=2}^{n} \lambda_{j} E_{j} e_{k}$. Note that if $d=0$, then $k=1, p_{11}=1$, and $p_{j 1}=0$ for $j=2, \ldots, n$, so that $E_{j} e_{k}=0$ for each such $j$. Hence, the first column on the right side of (1) is $m$. 
Suppose now that the claim holds for some $d \geq 0$, and that $d(1, k)=d+1$, say with $l \rightarrow k$ in $T$. From the fact that $-a_{k} e_{l}+e_{k}$ is an eigenvector for $M$ corresponding to $\lambda_{k}$, we find that $M e_{k}=a_{k} M e_{l}+\lambda_{k}\left(-a_{k} e_{l}+e_{k}\right)$. Since $d(1, l)=d$, we may apply the induction hypothesis to $M e_{l}$, and it now follows that $M e_{k}=a_{k} p_{1 l} m+a_{k} \sum_{j=2}^{n} \lambda_{j}\left(-a_{j} e_{i_{j}}+\right.$ $\left.e_{j}\right) p_{j l}+\lambda_{k}\left(-a_{k} e_{l}+e_{k}\right)=p_{1 k} m+\sum_{j=2, \ldots, n, j \hookrightarrow l} \lambda_{j}\left(-a_{j} e_{i_{j}}+e_{j}\right) p_{j k}+\lambda_{k}\left(-a_{k} e_{l}+e_{k}\right)$, where, if there is a path from $j$ to $l$ in $T$, we use the notation $j \hookrightarrow l$. Noting that the term $j=k$ does not appear in the sum above, since there is no path from $k$ to $l$ in $T$, and that $p_{k k}=1$, it now follows that $M e_{k}=p_{1 k} m+\sum_{j=2}^{n} \lambda_{j}\left(-a_{j} e_{i_{j}}+e_{j}\right) p_{j k}$, completing the proof of the induction step.

Observe that, using the notation of Proposition 4.3, since

$$
\left[\begin{array}{llll}
1 & p_{12} & \ldots & p_{1 n}
\end{array}\right]\left(-a_{j} e_{i_{j}}+e_{j}\right)=0
$$

for each $j=2, \ldots, n$, we find that $\left[\begin{array}{cccc}1 & p_{12} & \ldots & p_{1 n}\end{array}\right] m=1$. If we have a matrix $M$ satisfying (1), for each $j=2, \ldots, n$, we say that $\lambda_{j}$ is the eigenvalue associated with vertex $j$ of $T$.

We now apply Proposition 4.3 to discuss the relationships between the various parameters in (1) and the arborescence $T$ associated with $S$.

Proposition 4.4 Let $S$ be an $S^{2} N S$ sign pattern of order $n$ such that $S e_{1}=-\mathbf{1}$ and $S_{i i}=-1, i=1, \ldots, n$. Suppose that $e_{1}^{T} A^{-1}<0^{T}$ for each $A \in Q(S)$, and denote the arborescence associated with $S$ by $T$. Fix $A \in Q(S)$ and suppose that $A$ is in $S^{2} N S$ normalised form, with arc weights $a_{2}, \ldots, a_{n}$. Let $D=\left[\begin{array}{cccc}1 & & & \\ & \lambda_{2} & & \\ & & \ddots & \\ & & \lambda_{n}\end{array}\right]$ for some collection of real numbers $\lambda_{2}, \ldots, \lambda_{n}$, and suppose that $M$ is a nonnegative matrix such that $M A=A D$. Then the following conditions hold:

i) for each index $l=2, \ldots, n$ such that $1 \rightarrow l$ in $T, m_{1} \geq \lambda_{l}$;

ii) for each $j=2, \ldots, n, m_{j} p_{1 j}+\lambda_{j} \geq 0$;

iii) for each $j=2, \ldots, n$ and each $k$ such that $j \rightarrow k$ in $T, m_{j} p_{1 j}+\lambda_{j}-\lambda_{k} \geq 0$.

Proof: Since $M$ is a nonnegative matrix, in particular, $e_{1}^{T} M \geq 0^{T}$. Referring to (1), we then find that for each $l$ such that $1 \rightarrow l$ in $T$, we must have $m_{1} p_{1 l}-\lambda_{l} a_{l} p_{l l} \geq 0$. Since $p_{1 l}=a_{l}$ and $p_{l l}=1$, condition i) follows.

Next, consider an index $j$ between 2 and $n$. From (1) we have that $M_{j j}=m_{j} p_{1 j}+$ $e_{j}^{T} \sum_{l=2}^{n} \lambda_{l}\left(-a_{l} e_{i_{l}}+e_{l}\right) p_{l j}$. Note that if $j=i_{l}$ for some $l$, then $i_{l} \rightarrow j$ in $T$, and it follows that $p_{l j}=0$ then. Hence we find that $M_{j j}=m_{j} p_{1 j}+\lambda_{j} \geq 0$, so condition ii) follows. 
Finally suppose that $j=2, \ldots, n$ and that $j \rightarrow k$ for some index $k$. Referring to (1), we have $M_{j k}=m_{j} p_{1 k}+e_{j}^{T} \sum_{l=2}^{n} \lambda_{l}\left(-a_{l} e_{i_{l}}+e_{l}\right) p_{l k}$. Note that if $j \rightarrow l$ for some $l \neq k$, then $p_{l k}=0$, so it follows that $M_{j k}=m_{j} p_{1 k}+\lambda_{j} p_{j k}-\lambda_{k} a_{k} p_{k k}=$ $a_{k}\left(m_{j} p_{1 j}+\lambda_{j}-\lambda_{k}\right)$. Condition iii) now follows.

Remark 4.5 Suppose that $M$ is an $n \times n$ matrix having the form given in (1). An analysis similar to that in the proof of Proposition 4.4 yields the following conclusions: a) for an index $2 \leq k \leq n, M_{1 k}>0$ if and only if $m_{1}>\lambda_{l}$, where the path from 1 to $k$ in $T$ includes the arc $1 \rightarrow l$;

b) for distinct indices $i, k$ with $2 \leq i, k \leq n$, we have $M_{i k}>0$ if and only if either

- there is no path from $i$ to $k$ in $T$ and $m_{i}>0$, or

- there is a path from $i$ to $k$ in $T$, it includes the arc $i \rightarrow j$, and $m_{i} p_{1 i}+\lambda_{i}-\lambda_{j}>0$;

c) for an index $2 \leq i \leq n, M_{i i}>0$ if and only if $m_{i} p_{1 i}+\lambda_{i}>0$.

Here is one of the main results of this section.

Theorem 4.6 Let $S$ be an $S^{2} N S$ sign pattern as in Proposition 4.3 with underlying arborescence T. Let $\Lambda=\left\{\lambda_{2}, \ldots, \lambda_{n}\right\}$ be a multiset of real numbers. There is an $A \in Q(S)$, and a positive matrix $M$ that is diagonalised by $A$ and has spectrum $\Lambda \cup\{1\}$ if and only if $\max \left\{0, \lambda_{2}, \ldots, \lambda_{n}\right\}+\sum_{\lambda_{j}<0}\left|\lambda_{j}\right|<1$.

Proof: Suppose first that there is an $A \in Q(S)$, and a positive matrix $M$ such that $M A=A D$, where $D=\left[\begin{array}{cccc}1 & & & \\ & \lambda_{2} & & \\ & & \ddots & \\ & & & \lambda_{n}\end{array}\right]$; without loss of generality we take $A$

to be in $S^{2} N S$ normalised form. Adopting the notation of Proposition 4.3, it follows from Remark 4.5 that $m_{i}>0, i=1, \ldots, n, m_{i} p_{1 i}+\lambda_{i}>0$ for each $i=2, \ldots, n$, and for any arc $i \rightarrow j$ in $T, m_{i} p_{1 i}+\lambda_{i}-\lambda_{j}>0$.

If $\lambda_{j} \leq 0$ for each $j=2, \ldots, n$, then note that $1=m_{1}+\sum_{j=2}^{n} m_{j} p_{1 j}>$ $\sum_{\lambda_{j}<0} m_{j} p_{1 j} \geq \sum_{\lambda_{j}<0}\left|\lambda_{j}\right|$, as desired. Next, we consider the case that $\lambda_{j_{0}} \equiv$ $\max \left\{0, \lambda_{2}, \ldots, \lambda_{n}\right\}>0$. We label the path from 1 to $j_{0}$ in $T$ as $1 \rightarrow i_{1} \rightarrow i_{2} \rightarrow \ldots \rightarrow$ $i_{k-1} \rightarrow i_{k} \equiv j_{0}$. If $\lambda_{i_{l}} \geq 0$ for $l=1, \ldots k-1$, then from the fact that $m_{1}>\lambda_{i_{1}}$, and $m_{i_{l}} p_{1 i_{l}}+\lambda_{i_{l}}-\lambda_{i_{l+1}}>0$, for $l=1, \ldots k-1$, we find that $m_{1}+\sum_{l=1}^{k-1} m_{i_{l}} p_{1 i_{l}}>\lambda_{j_{0}}$. Also, for each $j$ such that $\lambda_{j}<0$, we have $m_{j} p_{1 j}>\left|\lambda_{j}\right|$. Consequently, we have $1=m_{1}+\sum_{j=2}^{n} m_{j} p_{1 j} \geq m_{1}+\sum_{l=1}^{k-1} m_{i_{l}} p_{1 i_{l}}+\sum_{\lambda_{j}<0} m_{j} p_{1 j}>\lambda_{j_{0}}+\sum_{\lambda_{j}<0}\left|\lambda_{j}\right|$. Finally, if some $\lambda_{i_{l}}<0$, then take $r$ as the index between 2 and $k-1$ such that $\lambda_{i_{r}}<0$, but $\lambda_{i_{s}} \geq 0$ for $s=r+1, \ldots, k-1$. As above, we have $\sum_{s=r}^{k-1} m_{i_{s}} p_{1 i_{s}}+\lambda_{i_{r}}-\lambda_{j_{0}}>0$. 
An argument analogous to the above now shows that $1=m_{1}+\sum_{j=2}^{n} m_{j} p_{1 j}>$ $\sum_{s=r}^{k-1} m_{i_{l}} p_{1 i_{l}}+\sum_{\lambda_{j}<0, j \neq i_{r}} m_{j} p_{1 j}>\lambda_{j_{0}}+\sum_{\lambda_{j}<0}\left|\lambda_{j}\right|$. Thus the desired inequality holds.

We now consider the converse, so suppose that $\max \left\{0, \lambda_{2}, \ldots, \lambda_{n}\right\}+\sum_{\lambda_{j}<0}\left|\lambda_{j}\right|<$ 1. For each vertex $j=2, \ldots, n$, we associate a corresponding element of $\Lambda$ as follows: for the vertices $l$ such that $1 \rightarrow l$, say $l_{1}, \ldots, l_{d}$, we associate the $d$ largest elements of $\Lambda$; thereafter, we associate the remaining elements of $\Lambda$ to vertices in $T$ in such a way that if $j \rightarrow k$ in $T$, then $\lambda_{j} \geq \lambda_{k}$.

Select a matrix $A \in Q(S)$. We first construct the column vector $\tilde{m}$ as follows: for each $j=2, \ldots, n$, such that $\lambda_{j} \geq 0$, set $\tilde{m}_{j}=0$; and for each $j=2, \ldots, n$, such that $\lambda_{j}<0$, we let $\tilde{m}_{j}=\frac{\left|\lambda_{j}\right|}{p_{1 j}}$. Now set $\tilde{m}_{1}$ equal to $1-\sum_{j=2}^{n} \tilde{m}_{j} p_{1 j}$, and note that $\tilde{m}_{1}=\sum_{\lambda_{j}<0}\left|\lambda_{j}\right|>\max \left\{0, \lambda_{2}, \ldots, \lambda_{n}\right\}$. Since this last inequality is strict, it now follows that we can find a column vector $m$ such that $m_{j}>0$ for each $j$ such that $\lambda_{j} \geq 0, m_{j}>\frac{\left|\lambda_{j}\right|}{p_{1 j}}$ for each $j$ such that $\lambda_{j}<0$, and $m_{1}=1-\sum_{j=2}^{n} m_{j} p_{1 j}>$ $\max \left\{0, \lambda_{2}, \ldots, \lambda_{n}\right\}$. With this choice of the column vector $m$, and this association between the elements of $\Lambda$ and the vertices $2, \ldots, n$ of $T$, we let $M$ be given by (1). Referring to Remark 4.5, and recalling that if $i \rightarrow j$ in $T$, then $\lambda_{i} \geq \lambda_{j}$, it now follows that $M$ is a positive matrix.

From (1) we find readily that $\left[\begin{array}{llll}1 & p_{12} & \ldots & p_{1 n}\end{array}\right]$ is a positive left eigenvector for $M$ corresponding to the eigenvalue 1 . Hence 1 is the Perron value for $M$. Fix an index $j=2, \ldots, n$. For each index $l \neq j$ between 1 and $n$, we have

$$
\left[\begin{array}{llll}
p_{l 1} & p_{l 2} & \ldots & p_{l n}
\end{array}\right]\left(-a_{j} e_{i_{j}}+e_{j}\right)=-a_{j} p_{l i_{j}}+p_{l j}=0,
$$

while

$$
\left[\begin{array}{llll}
p_{j 1} & p_{j 2} & \ldots & p_{j n}
\end{array}\right]\left(-a_{j} e_{i_{j}}+e_{j}\right)=-a_{j} p_{j i_{j}}+p_{j j} e_{j}=0+1=1 .
$$

It now follows that for each $j=2, \ldots, n,-a_{j} e_{i_{j}}+e_{j}$ is an eigenvector of $M$ corresponding to eigenvalue $\lambda_{j}$. Thus the spectrum of $M$ is given by $\Lambda \cup\{1\}$.

Finally, we let $\tilde{A}$ be formed from $A$ by replacing its first column by an all negative eigenvector of $M$ corresponding to its Perron value 1 , we see that $\tilde{A} \in Q(S)$ and diagonalises $M$.

Remark 4.7 A minor modification of the argument in Theorem 4.6 shows that given an $S^{2} N S$ sign pattern $S$ as in Proposition 4.3, and a multiset of real numbers $\Lambda=\left\{\lambda_{2}, \ldots, \lambda_{n}\right\}$, there is an $A \in Q(S)$, and a nonnegative matrix $M$ such that $A$ diagonalises $M$ and $M$ has spectrum $\Lambda \cup\{1\}$ only if $\max \left\{0, \lambda_{2}, \ldots, \lambda_{n}\right\}+\sum_{\lambda_{j}<0}\left|\lambda_{j}\right| \leq 1$. 
In the next sequence of results, we consider a multiset of real numbers $\Lambda=$ $\left\{\lambda_{2}, \ldots, \lambda_{n}\right\}$ such that $\max \left\{0, \lambda_{2}, \ldots, \lambda_{n}\right\}+\sum_{\lambda_{j}<0}\left|\lambda_{j}\right|=1$. Observe that if $\Lambda \cup\{1\}$ is the spectrum of an irreducible nonnegative matrix of order $n$, then necessarily $\Lambda$ must contain at least one negative element. We begin our analysis with the case that $\Lambda$ contains no positive elements.

Proposition 4.8 Suppose that we have a multiset of real numbers $\Lambda=\left\{\lambda_{2}, \ldots, \lambda_{n}\right\}$, such that $\lambda_{j} \leq 0, j=2, \ldots, n$, and such that $\sum_{\lambda_{j}<0}\left|\lambda_{j}\right|=1$. Denote the number of negative $\lambda_{j}$ s by $\nu$. There is an $S^{2} N S$ sign pattern matrix $S$, a matrix $A \in Q(S)$, and an irreducible nonnegative matrix $M$ with spectrum $\{1\} \cup \Lambda$ that is diagonalised by $A$ if and only if $\nu \geq \frac{n}{2}$.

Proof: Suppose that there is an irreducible nonnegative matrix $M$ with the desired properties. Take $M$ to be in the form (1), and for each $j=2, \ldots, n$, let $\lambda_{j}$ be associated with vertex $j$ in the arborescence $T$ associated with $S$. From Proposition 4.4 and the hypotheses on $\Lambda$, we have $1=m_{1}+\sum_{j=2}^{n} m_{j} p_{1 j} \geq m_{1}+\sum_{\lambda_{j}<0}\left|\lambda_{j}\right|+$ $\sum_{\lambda_{j}=0} m_{j} p_{1 j} \geq \sum_{\lambda_{j}<0}\left|\lambda_{j}\right|=1$. We deduce that $m_{1}=0$, and that $m_{j}=0$ for any $j$ such that $\lambda_{j}=0$. From Remark 4.5 we find that for each $j$ such that $\lambda_{j}=0$, there is an index $l$ such that $j \rightarrow l$ in $T$ and $\lambda_{l}<0$; similarly, there is an index $l$ such that $1 \rightarrow l$ in $T$ and $\lambda_{l}<0$. Since vertices $2, \ldots, n$ of $T$ all have indegree 1 , we find that $\left|\left\{\lambda_{j} \mid \lambda_{j}<0\right\}\right| \geq 1+\left|\left\{\lambda_{j} \mid \lambda_{j}=0\right\}\right|$, or equivalently, $\nu \geq 1+n-1-\nu$. Hence $\nu \geq \frac{n}{2}$.

Conversely, suppose that $\nu \geq \frac{n}{2}$, so that $\nu \geq 1+n-1-\nu$. First we consider the case that $\lambda_{j}<0, j=2, \ldots, n$ and $\sum_{j=2}^{n}\left|\lambda_{j}\right|=1$. Let $M$ be given by

$$
M=\left[\begin{array}{ccccc}
0 & \left|\lambda_{2}\right| & \left|\lambda_{3}\right| & \ldots & \left|\lambda_{n}\right| \\
\left|\lambda_{2}\right| & 0 & \left|\lambda_{2}\right| & \ldots & \left|\lambda_{2}\right| \\
\left|\lambda_{3}\right| & \left|\lambda_{3}\right| & 0 & \ldots & \left|\lambda_{3}\right| \\
\vdots & & & \ddots & \vdots \\
\left|\lambda_{n}\right| & \left|\lambda_{n}\right| & \ldots & \left|\lambda_{n}\right| & 0
\end{array}\right] .
$$

Observe that $M$ is nonnegative and irreducible, and that each column sum of $M$ is 1 ; hence the Perron value of $M$ is 1 . Further, for each $j=2, \ldots, n$, we have $M\left(e_{1}-e_{j}\right)=\lambda_{j}\left(e_{1}-e_{j}\right)$. It now follows readily that the spectrum of $M$ is $\{1\} \cup \Lambda$, and that $M$ is diagonalised by the matrix $A$ whose first column is a right Perron vector of $M$ (scaled to have all negative entries), and whose remaining columns are $e_{1}-e_{j}, j=2, \ldots, n$. It is straightforward to determine that there is an $S^{2} N S$ sign pattern matrix $S$ such that $A \in Q(S)$.

Next, we consider the case that some of the $\lambda_{j}$ s are equal to zero. For concreteness we assume that $\lambda_{2}=\ldots=\lambda_{n-\nu}=0$, and that $\lambda_{j}<0$, for $j=n-\nu+1, \ldots, n$. Consider the arborescence $T$ on vertices $1, \ldots, n$ with the following arcs: $1 \rightarrow j, j=$ 
$2, \ldots, n-\nu+1 ; i \rightarrow i+n-\nu, i=2, \ldots, n-\nu ;$ and $n-\nu+1 \rightarrow j, j=2 n-2 \nu+1, \ldots, n$. Next, we set $a_{j}=1, j=2, \ldots, n$, set $m_{j}=0, j=1, \ldots, n-\nu$, and set $m_{j}=\left|\lambda_{j}\right|, j=$ $n-\nu+1, \ldots, n$. Now we let $M$ be given by (1). Appealing to Remark 4.5, we see that rows $n-\nu+2, \ldots, n$ of $M$ have all positive entries off the diagonal, that $M_{1 n}>0$, and that for each $j=2, \ldots, n-\nu+1, M_{j, j+n-\nu}>0$. It now follows that $M$ is irreducible and nonnegative. Let $S$ be the $S^{2} N S$ sign pattern associated with $T$, (where the entries in the first column and diagonal of $S$ all -1$)$, and let $A$ be the $(0,1,-1)$ matrix in $Q(S)$. As in the proof of Theorem 4.6, we find from (1) we find that for each $j=2, \ldots, n, A e_{j}$ is an eigenvector for $M$ corresponding to eigenvalue $\lambda_{j}$, and that the Perron value for $M$ is 1 . Hence $M$ has spectrum $\{1\} \cup \Lambda$ and it follows that $M$ is diagonalised by a matrix in $Q(S)$.

The following technical result will be useful in the sequel.

Lemma 4.9 Suppose that we have disjoint sets $S_{1}, \ldots, S_{k+1}$, of cardinalities $\mu_{1}, \ldots$, $\mu_{k+1}$, respectively. For each $j=1, \ldots, k+1$, and each element $s \in S_{j}$, we define $v(s)=j$. We have $\mu_{k+1} \geq \max \left\{\mu_{1}, \ldots, \mu_{k}\right\}$ if and only if there is a directed graph $D$ whose vertices are indexed by the elements of $\cup_{i=1}^{k+1} S_{i}$, such that each vertex in $\cup_{i=2}^{k+1} S_{i}$ has indegree 1 , and for any vertex $j \in \cup_{i=1}^{k} S_{i}$, there is a vertex $l$ such that $j \rightarrow l$ and $v(j)>v(l)$.

Proof: Suppose that such a directed graph $D$ exists. We claim by induction on $i$ that $\mu_{k+1} \geq \mu_{k-i+1}$. For $i=1$, we note that for each vertex $s \in S_{k}$, there is a vertex $t \in S_{k+1}$ such that $s \rightarrow t$. Since each vertex in $S_{k+1}$ has indegree 1 , it now follows that $\mu_{k} \leq \mu_{k+1}$. Next we suppose that the claim holds for $i=k-l$, and consider the vertices in $D$ indexed by the elements of $\cup_{i=l}^{k+1} S_{i}$. For each $l \leq p, q \leq k+1$ with $p<q$, let $\gamma_{p, q}$ be the number of vertices in $S_{p}$ with outarcs out to vertices in $S_{q}$. Then $\mu_{p}=\sum_{q=p+1}^{k+1} \gamma_{p, q}$. Note also that

$$
\mu_{k+1} \geq \sum_{p=l}^{k} \gamma_{p, k+1} .
$$

From (2), we find that $\mu_{k+1} \geq \sum_{p=l}^{k+1}\left(\mu_{p}-\sum_{q=p+1}^{k} \gamma_{p, q}\right)+\mu_{k}=\mu_{l}+\sum_{j=l+1}^{k}\left(\mu_{j}-\right.$ $\left.\sum_{p=l}^{j-1} \gamma_{p, j}\right) \geq \mu_{l}$. Hence $\mu_{k+1} \geq \mu_{l}$, which completes the proof of the induction step. We conclude that $\mu_{k+1} \geq \max \left\{\mu_{1}, \ldots, \mu_{k}\right\}$.

To prove the converse, we proceed by induction on $\mu_{k+1}$. If $\mu_{k+1}=1$, then necessarily $\mu_{j}=1$ for $j=1, \ldots, k+1$. For each $j=1, \ldots, k+1$, let $s_{j}$ denote the single element in $S_{j}$; the directed graph $s_{1} \rightarrow s_{2} \rightarrow \ldots \rightarrow s_{k+1}$ has the desired properties. Suppose that the conclusion holds for some $p \geq 1$, and that $\mu_{k+1}=p+1$. For each 
$j=1, \ldots, k+1$, select an element $s_{j} \in S_{j}$, and construct the directed path $P$ given by $s_{1} \rightarrow s_{2} \rightarrow \ldots \rightarrow s_{k+1}$. Next, we consider the sets $S_{j} \backslash\left\{s_{j}\right\}, j=1, \ldots, k+1$, and note that their cardinalities are $\mu_{j}-1, j=1, \ldots, k+1$. Thus, the induction hypothesis applies, so there is a directed graph $\tilde{D}$ with such that each vertex in $\cup_{i=2}^{k+1}\left(S_{i} \backslash s_{i}\right)$ has indegree 1 , and for any vertex $j \in \cup_{i=1}^{k}\left(S_{i} \backslash s_{i}\right)$, there is a vertex $l$ such that $j \rightarrow l$ and $v(j)>v(l)$. The directed graph $D=P \cup \tilde{D}$ now has the desired properties.

Our next result provides a necessary condition in order that $\Lambda$ is realised as the spectrum of an irreducible nonnegative matrix that is diagonalised by an $A \in Q(S)$ when $S$ is an $S^{2} N S$ sign pattern.

Proposition 4.10 Suppose that we have a multiset of real numbers $\Lambda=\left\{\lambda_{2}, \ldots, \lambda_{n}\right\}$, with $\max \left\{\lambda_{2}, \ldots, \lambda_{n}\right\}>0$. Partition $\Lambda$ as $\cup_{j=1}^{k+1} S_{j}$ so that all negative elements of $\Lambda$ are in $S_{k+1}$, and so that for each $j=1, \ldots, k$, all elements of $S_{j}$ are equal, and for any $i, j=1, \ldots, n$, if $s \in S_{i}, t \in S_{j}$ and $i<j$, then $s>t$. For each $j=1, \ldots, k+1$, denote the cardinality of $S_{j}$ by $\mu_{j}$.

Suppose that $\max \left\{\lambda_{2}, \ldots, \lambda_{n}\right\}+\sum_{\lambda_{j}<0}\left|\lambda_{j}\right|=1$, and that there is an $S^{2} N S$ sign pattern $S$, a matrix $A \in Q(S)$, and an irreducible nonnegative matrix $M$ that is diagonalised by $A$ and has spectrum $\Lambda \cup\{1\}$. Then $\mu_{k+1} \geq \max \left\{\mu_{1}, \mu_{2}-1, \ldots, \mu_{k-1}-\right.$ $\left.1, \mu_{k}\right\}$.

Proof: We begin by noting that if the distinct nonnegative elements of $\Lambda$ are $\lambda_{m_{1}}>$ $\ldots>\lambda_{m_{k}}$, then they have multiplicities $\mu_{1}, \ldots, \mu_{k}$, respectively; further, for each $j=1, \ldots, k, S_{j}=\left\{\lambda_{m_{j}}^{\left(\mu_{j}\right)}\right\}$, while $S_{k+1}$ is the multiset consisting of the negative elements of $\Lambda$.

Let $T$ be the arborescence associated with $S$, assume without loss of generality that $A$ is in $S^{2} N S$ normalised form, and let the arc weights be $a_{j}, j=2, \ldots, n$. We take $M$ to be in the form (1). Let $\hat{\lambda}=\max \left\{\lambda_{2}, \ldots, \lambda_{n}\right\}$. Suppose that $T$ contains the path $1 \rightarrow i_{1} \rightarrow \ldots \rightarrow i_{q}$ where $\lambda_{i_{q}}=\hat{\lambda}$. As in the proof of Theorem 4.6, there are two cases to consider. Either

a) $\lambda_{i_{l}} \geq 0$ for each $l=1, \ldots, q$; or

b) there is some index $r$ such that $\lambda_{i_{r}}<0$ and $\lambda_{i_{s}} \geq 0, s=r+1, \ldots, q$.

Suppose that a) holds. We have

$$
\begin{array}{r}
1=m_{1}+\sum_{l=1}^{q-1} m_{i_{l}} p_{1 i_{l}}+\sum_{\lambda_{j}<0} m_{j} p_{1 j}+\sum_{\lambda_{j} \geq 0, j \neq i_{1}, \ldots, i_{q-1}} m_{j} p_{1 j} \geq \\
\hat{\lambda}+\sum_{\lambda_{j}<0}\left|\lambda_{j}\right|+\sum_{\lambda_{j} \geq 0, j \neq i_{1}, \ldots, i_{q-1}} m_{j} p_{1 j} \geq 1 .
\end{array}
$$


Necessarily, it must be the case that $m_{j} p_{1 j}=\left|\lambda_{j}\right|$ if $\lambda_{j}<0, m_{j}=0$ if $\lambda_{j} \geq 0$ and $j \neq i_{1}, \ldots, i_{q-1}, m_{1}=\lambda_{i_{1}}$, and $m_{i_{l}} p_{1 i_{l}}=\lambda_{i_{l+1}}-\lambda_{i_{l}}, l=1, \ldots, q-1$. In particular, we find that for each $j \geq 2$ such that $\lambda_{j} \geq 0$ and $j \neq i_{1}, \ldots, i_{q-1}$ we have $m_{j}=0$, and for each $l$ such that $\lambda_{i_{l+1}}=\lambda_{i_{l}} \geq 0$, we have $m_{i_{l}}=0$. Now, for each $j=2, \ldots, k+1$, let $\tilde{\mu}_{j}=\mu_{j}-1$ if, for some $1 \leq l \leq q-1, \lambda_{i_{l}} \in S_{j}$ and $\lambda_{i_{l+1}}>\lambda_{i_{l}} \geq 0$, and set $\tilde{\mu}_{j}=\mu_{j}$ otherwise. Suppose that in $T$ we have $1 \rightarrow l$ for some $l$ such that $\lambda_{l}<0$. In that case, we consider the directed subgraph of $T$ induced by the vertices $j \geq 2$ such that $m_{j}=0$, as well as the vertices $i \neq l$ such that $\lambda_{i}<0$. That subgraph is partitioned as in Lemma 4.9 into subsets of cardinalities $\mu_{1}, \tilde{\mu}_{2}, \ldots, \tilde{\mu}_{k}, \mu_{k+1}-1$. By Lemma 4.9 we thus find that $\mu_{k+1}-1 \geq \max \left\{\mu_{1}, \tilde{\mu}_{2}, \ldots, \tilde{\mu}_{k}\right\} \geq \max \left\{\mu_{1} \mu_{2}-1, \ldots, \mu_{k}-1\right\}$; the desired inequality now follows. On the other hand, if $1 \rightarrow p$ only for $\lambda_{p} \geq 0$, then since $M$ is irreducible, it follows from Remark 4.5 that $\lambda_{i_{1}} \notin S_{k}$, so that $\tilde{\mu}_{k}=\mu_{k}$. Again consider the directed subgraph of $T$ induced by the vertices $j \geq 2$ such that $m_{j}=0$, as well as the vertices $i \neq l$ such that $\lambda_{i}<0$. In this case, the subgraph is partitioned into subsets of cardinalities $\mu_{1}, \tilde{\mu}_{2}, \ldots, \tilde{\mu}_{k}, \mu_{k+1}$, and from Lemma 4.9 we find that $\mu_{k+1} \geq \max \left\{\mu_{1}, \tilde{\mu}_{2}, \ldots, \tilde{\mu}_{k}\right\} \geq \max \left\{\mu_{1}, \mu_{2}-1, \ldots, \mu_{k-1}-1, \mu_{k}\right\}$, as desired.

Next we suppose that b) holds. We then have $1=m_{1}+\sum_{l=r}^{q-1} m_{1 i_{l}} p_{1 i_{l}}+\sum_{\lambda_{j}<0} p_{1 j}+$ $\sum_{\lambda_{j} \geq 0, j \neq i_{r}, \ldots, i_{q-1}} m_{j} p_{1 j} \geq m_{1}+\hat{\lambda}+\sum_{\lambda_{j}<0}\left|\lambda_{j}\right|+\sum_{\lambda_{j} \geq 0, j \neq i_{r}, \ldots, i_{q-1}} m_{j} p_{1 j} \geq 1$. We deduce that $m_{1}=0, m_{i_{l}} p_{1 i_{l}}=\lambda_{i_{l+1}}-\lambda_{i_{l}}, l=r+1, \ldots, q-1, m_{j} p_{1 j}=\left|\lambda_{j}\right|$ whenever $\lambda_{j}<0$, and $m_{j}=0$ for any $j$ such that $\lambda_{j} \geq 0$ and $j \neq i_{r+1}, \ldots, i_{q-1}$. Since $m_{1}=0$, there is an index $l$ such that $1 \rightarrow l$ and $\lambda_{l}<0$. Arguing as in case a), it now follows that $\mu_{k+1}-1 \geq \max \left\{\mu_{1}, \mu_{2}-1, \ldots, \mu_{k}-1\right\}$.

The lemma below will be useful in establishing one of the main results of this section.

Lemma 4.11 Suppose that we have an $n \times n S^{2} N S$ sign pattern $S$ with associated arborescence $T$, a matrix $A \in Q(S)$ in $S^{2} N S$ normalised form, a multiset $\Lambda=\left\{\lambda_{2}, \ldots, \lambda_{n}\right\}$, and a nonnegative matrix $M$ satisfying (1). Suppose further that

i) $m_{1}>\lambda_{l}$ for some index $l$ such that $1 \rightarrow l$ in $T$, and

ii) for each $j=2, \ldots, n$, if $m_{j}=0$, then there is an index $l$ such that $\lambda_{j}>\lambda_{l}$ and $j \rightarrow l$ in $T$.

Then $M$ is irreducible.

Proof: We begin by noting that from ii), we find that if $k$ is a pendant vertex of $T$, then necessarily $m_{k}>0$; referring to Remark 4.5 we see that there is an arc from $k$ to any other vertex of $D(M)$. From i), we see that in $D(M), 1 \rightarrow k$ for some vertex $k$ corresponding to a pendant vertex of $T$, and similarly, for any index $j$ such that $m_{j}=0$, there is a vertex $k$ that is pendant in $T$ and such that $j \rightarrow k$ in $D(M)$. 
Finally, for any $j$ such that $m_{j}>0$, we have the arc $j \rightarrow 1$ in $D(M)$. It now follows readily that $M$ is irreducible.

The next result provides a converse to Proposition 4.10 in the case that $k \geq 2$.

Theorem 4.12 Suppose that we have a multiset of real numbers $\Lambda=\left\{\lambda_{2}, \ldots, \lambda_{n}\right\}$, with $\max \left\{\lambda_{2}, \ldots, \lambda_{n}\right\}>0$. Partition $\Lambda$ as $\cup_{j=1}^{k+1} S_{j}$ so that all negative elements of $\Lambda$ are in $S_{k+1}$, and so that for each $j=1, \ldots, k$, all elements of $S_{j}$ are equal, and for any $i, j=1, \ldots, n$, if $s \in S_{i}, t \in S_{j}$ and $i<j$, then $s>t$. For each $j=1, \ldots, k+1$, denote the cardinality of $S_{j}$ by $\mu_{j}$. Assume that $k \geq 2$.

Suppose that $\max \left\{\lambda_{2}, \ldots, \lambda_{n}\right\}+\sum_{\lambda_{j}<0}\left|\lambda_{j}\right|=1$, and that $\mu_{k+1} \geq \max \left\{\mu_{1}, \mu_{2}-\right.$ $\left.1, \ldots, \mu_{k-1}-1, \mu_{k}\right\}$. Then there is an $S^{2} N S$ sign pattern $S$, a matrix $A \in Q(S)$, and an irreducible nonnegative matrix $M$ that is diagonalised by $A$ and has spectrum $\{1\} \cup \Lambda$.

Proof: For each $j=1, \ldots, k$, let $x_{j}$ denote the common value of the elements in $S_{j}$. We now construct an arborescence $T$ as follows. Begin with the $\operatorname{arcs} 1 \rightarrow 2$ and $1 \rightarrow 3$, as well as $i \rightarrow i+1, i=3, \ldots, k+1$. For each such $i=2, \ldots, k+1$, associate the vertex $i$ with the value $x_{k-i+2}$. Now, using Lemma 4.9 and the hypothesis that $\mu_{k+1} \geq \max \left\{\mu_{1}, \mu_{2}-1, \ldots, \mu_{k-1}-1, \mu_{k}\right\}$, we may complete this collection of arcs to an arborescence $T$ such that there is a directed path from 1 to any vertex of $T$, each vertex $i=2, \ldots, n$ is associated with an element of $\Lambda$, every pendant vertex is associated with an element in $S_{k+1}$ and conversely, $2 \rightarrow l$ for some $l$ such that $\lambda_{l}<0$, and for any $i \geq k+1$, there is an index $l$ such that $i \rightarrow l$ and $\lambda_{i}>\lambda_{l}$. Set $m_{1}=x_{k-1}$, $m_{i}=x_{k-i+1}-x_{k-i+2}, i=3, \ldots, k, m_{j}=\left|\lambda_{j}\right|$ for each $j$ such that $\lambda_{j}<0$, and all remaining $m_{j}$ s equal to 0 .

Set $a_{j}=1, j=2, \ldots, n$, let $S$ denote the $(0,1,-1) S^{2} N S$ sign pattern with associated arborescence $T$ and negative first column, and suppose that $A \in Q(S)$ is in $S^{2} N S$ normalised form. Let $M$ be given by (1). From Remark 4.5 and Lemma 4.11, we find that $M$ is irreducible and nonnegative; as in the proof of Theorem 4.6, we find that $M$ has spectrum $\Lambda \cup\{1\}$. Further, the vectors $A e_{i}, i=2, \ldots, n$, are eigenvectors for $M$. Letting $v$ denote a negative Perron vector for $M$, we find that the matrix $\tilde{A}$ formed from $A$ by replacing its first column by $v$ is in $Q(S)$ and diagonalises $M$.

Remark 4.13 A mild modification of the arguments in Proposition 4.10 and Theorem 4.12 shows the following: 
Suppose that we have a multiset of real numbers $\Lambda=\left\{\lambda_{2}, \ldots, \lambda_{n}\right\}$, with $\max \left\{\lambda_{2}, \ldots, \lambda_{n}\right\}$ $>0$, such that

$$
\max \left\{\lambda_{2}, \ldots, \lambda_{n}\right\}+\sum_{\lambda_{j}<0}\left|\lambda_{j}\right|=1 .
$$

Suppose further that $\Lambda$ has just one distinct positive element, of multiplicity $\mu_{1}$, and $\mu_{2} \equiv n-1-\mu_{1}$ negative elements. Then there is an $S^{2} N S$ sign pattern $S$, a matrix $A \in Q(S)$, and an irreducible nonnegative matrix $M$ that is diagonalised by $A$ and has spectrum $\{1\} \cup \Lambda$ if and only if $\mu_{2} \geq \mu_{1}+1$.

Remark 4.14 Let $\Lambda=\left\{\lambda_{2}, \ldots, \lambda_{n}\right\}$ be a multiset of real numbers such that

$$
\max \left\{\lambda_{2}, \ldots, \lambda_{n}\right\}+\sum_{\lambda_{j}<0}\left|\lambda_{j}\right|=1 .
$$

Taken together, Propositions 4.8 and 4.10, Theorem 4.12 and Remark 4.13 provide necessary and sufficient conditions in order that there is an $S^{2} N S$ sign pattern $S$, a matrix $A \in Q(S)$, and an irreducible nonnegative matrix $M$ that is diagonalised by $A$ and has spectrum $\{1\} \cup \Lambda$. Proposition 4.8 covers the case that $\Lambda$ contains only negative elements, while Proposition 4.8 and Remark 4.13 cover the case that $\Lambda$ contains just one nonnegative element (which may have multiplicity greater than 1). Finally, Proposition 4.10 and Theorem 4.12 cover the case that $\Lambda$ contains two or more distinct nonnegative elements.

Acknowledgment: The author thanks Annalisa Zappavigna for a conversation which initiated this investigation. The author is very grateful to the referee, whose insightful comments resulted in substantial improvements to the paper.

\section{References}

[1] A. Borobia, Inverse eigenvalue problems, in Handbook of Linear Algebra, L. Hogben, ed., Chapman and Hall, Boca Raton, 2007, 20-1 - 20-14.

[2] R.A. Brualdi and H. Ryser, Combinatorial Matrix Theory, Cambridge University Press, Cambridge, 1991.

[3] R.A. Brualdi and B.L. Shader, Matrices of Sign-Solvable Linear Systems, Cambridge University Press, Cambridge, 1995.

[4] N. Dmitriev and E. Dynkin, On characteristic roots of stochastic matrices, Bull. Acad. Sci. URSS. Sr. Math. 10, (1946), 167-184. 
[5] P. Egleston, T. Lenker and S. Narayan, The nonnegative inverse eigenvalue problem, Linear Algebra Appl. 379 (2004), 475-490.

[6] F.I. Karpelevich, On characteristic roots of matrices with nonnegative coefficients, Uspehi Matem. Nauk (N.S.) 4, (1949) 177-178.

[7] E. Seneta, Non-negative Matrices and Markov Chains, Springer-Verlag, New York, 1981. 